\title{
Down-regulation of microRNA-10a mediates the anti-tumor effect of icaritin in A549 cells via the PTEN/AKT and ERK pathway
}

\author{
Xiangdong Lu ${ }^{1, \star}$, Beiyun Xue ${ }^{2, \star}$, Tingrong Zhang ${ }^{1}$, Xie Zhou ${ }^{1}$ and Yao Zhang ${ }^{1}$ \\ ${ }^{1}$ Department of Oncology, The Affiliated Jiangyin Hospital of Southeast University Medical College, Jiangyin, China \\ ${ }^{2}$ Department of Traditional Chinese Medicine, The Affiliated Jiangyin Hospital of Southeast University Medical College, \\ Jiangyin, China
}

\begin{abstract}
Icaritin, a prenylflavonoid derivative from Epimedium Genus, has been reported to exhibit tumor inhibitory effects on many types of tumor cells. Numerous studies have demonstrated that microRNAs (miRs) involve in the biological process of carcinogenesis by controlling expression of their target mRNAs to facilitate tumor growth, invasion, angiogenesis, and immune evasion. miR-124 was reported to involve in the icaritin-induced mitochondrial apoptosis in human carcinoma cells. However, the roles of other miRs in the anti-tumor effects of icaritin and its underlying mechanisms still need to be elucidated. In the present study, realtime-PCR results showed that miR-10a was significantly down-regulated after icaritin treatment in human non-small cell lung cancer cells (A549). Over-expression of miR-10a in A549 cells dramatically abrogated the anti-tumor effects of icaritin on cell proliferation, apoptosis, migration, while suppression of miR-10a partially reproduced the anti-tumor effects of icaritin. Furthermore, we found that the regulation of miR-10a in the anti-tumor effects of icaritin was mediated via the PTEN/AKT/ERK pathway by directly targeting to PTEN. Taken together, miR-10a targets PTEN to mediate the anti-tumor effect of icaritin in A549 cells, which provides a novel insight into the anti-tumor mechanism of icaritin and may provide a new strategy for lung cancer therapy.
\end{abstract}

Key words: Icaritin - miR-10a - PTEN - A549 cells — Lung cancer

\section{Introduction}

As one of the most common malignant tumors, lung cancer becomes a leading cause of cancer-related deaths in the world (Torre et al. 2015; Bray et al. 2018). Non-small-cell lung cancer (NSCLC) is the primary type of lung cancer, which accounts for about $85 \%$ of all the lung cancer cases (Molina et al. 2008). Some NSCLCs are reported to be resistant to chemotherapy and radiation therapy, and grow and migrate quickly (Buttery et al. 2004; Caramori et al. 2011). Although numerous efforts have been made in the diagnosis and treatment of NSCLC, including chemotherapy,

\footnotetext{
* These authors contributed equally to this work.

Correspondence to: Yao Zhang, Department of Oncology, The Affiliated Jiangyin Hospital of Southeast University Medical College, No. 163 Shoushan Road, Jiangyin, Wuxi, Jiangsu Province, 214400, China

E-mail: zhangyaojy@126.com
}

radiotherapy, stem cell transplantation, and immunotherapy (Zappa and Mousa 2016; Cho 2017), the 5-year survival rate of NSCLC patients remains very low, which is still less than $15 \%$ (Molina et al. 2008). One of the challenges in the treatment of NSCLC patients is the metastasis to other organs through the whole body (Zappa and Mousa 2016). It has been reported that the tumor metastasis is a complex process involving in many factors (Seyfried and Huysentruyt 2013; Liu et al. 2017). So far, the molecular mechanisms underlying the tumor metastasis remain unknown. However, some anticancer agents that can effectively inhibit and prevent tumor metastasis have been demonstrated to be beneficial for the treatment of NSCLC (Lin et al. 2009; Sun and Gao 2018; Wang et al. 2018).

Icaritin is a prenylflavonoid derivative from a traditional Chinese herb, Epimedium Genus. Recently, accumulating studies have demonstrated an anti-tumor effect of icaritin in various cancers, including solid tumors and hematological cancers, such as lung cancer (Zheng et al. 2014), hepatocel- 
lular carcinoma (Sun et al. 2015; Hu et al. 2016; Lu et al. 2017), breast cancer (Guo et al. 2011; Tiong et al. 2012), esophageal cancer (Han et al. 2018), glioblastoma (Li et al. 2018; Liu et al. 2018), leukemia and lymphoma (Zhu et al. 2011; Li et al. 2013; Li et al. 2014; Wu et al. 2015; Zhu et al. 2015; Yang et al. 2019). Icaritin exerts its anti-tumor effects in various cancers mainly by inhibiting cell proliferation, inducing cell differentiation and apoptosis, suppressing cell migration and invasion (Zheng et al. 2014; Xu et al. 2015). MicroRNAs (miRs) are known to involve in the biological process of carcinogenesis by controlling expression of their target mRNAs to facilitate tumor growth, invasion, angiogenesis, and immune evasion (Hayes et al. 2014; Peng and Croce 2016). However, whether miRs involve in the inhibitory effects of icaritin against tumor growth and metastasis is largely unknown, although miR-124 was reported to involve in the icaritin-induced mitochondrial apoptosis in human carcinoma cells (Jin et al. 2017).

It is reported that in human NSCLC A549 cells, icaritin inhibit cancer cell growth by inducing cell cycle arrest at S phase and promote cell apoptosis (Zheng et al. 2014). In the present study, we found that the inhibitory effect of icaritin in A549 cells well correlated with the down-regulation of miR10a. Futhermore, miR-10a targets to PTEN to mediate the anti-tumor effects of icaritin in the proliferation, apoptosis, migration of A549 cells.

\section{Material and Methods}

\section{Cell culture and treatment}

Human lung cancer cell line A549 was purchased from ATCC $\left(\right.$ ATCC ${ }^{\circledast}$ CCL- $185^{\mathrm{TM}}$ ), and cultured in DMEM medium supplemented with $10 \%$ fetal bovine serum (Invitrogen), $100 \mathrm{u} / \mathrm{ml}$ penicillin (Sigma-Aldrich), and $100 \mathrm{ug} / \mathrm{ml}$ streptomycin (Sigma-Aldrich) at $37^{\circ} \mathrm{C}$ in a humidity incubator with $5 \% \mathrm{CO}_{2}$ and $95 \%$ air.

Cells were plated in 6-wells or 96-well plates in different experiments. Different concentrations of icaritin $(5,10,20$, 40 and $80 \mathrm{mM}$ ) were added into the medium in this study. After $24 \mathrm{~h}$ or $48 \mathrm{~h}$ incubation, the cells were collected to detect cell proliferation, apoptosis, migration and invasion.

\section{MTT assay}

The level of cell growth and proliferation were determined by using MTT assay kit (Abcam, ab211091) according to the manufacturer's instructions. Briefly, A549 cells were seeded in 96-well plates. After treatment, serum-containing media was replaced with serum-free media and MTT reagent in cell cultures, then incubated at $37^{\circ} \mathrm{C}$ for $3 \mathrm{~h}$. After incubation, MTT solvent was added, and incubated for $15 \mathrm{~min}$. The cell viability was determined by the measured absorbance at OD $590 \mathrm{~nm}$.

\section{Annexin-PI staining and flow cytometry}

After different treatments, cell apoptosis was examined by propidium iodide (PI) and fluorescein isothiocynate (FITC)conjugated Annexin V double staining and flow cytometry analysis according to the manufacturer's instructions. Briefly, A549 cells was seeded into 24-wells plate. After icaritin treatment, cells were washed with PBS and re-suspended in binding buffer containing FITC-labeled Annexin V and PI for $15 \mathrm{~min}$ at room temperature in the dark. After washing twice with PBS, the rate of apoptotic cells was measured by flow cytometry analysis.

\section{Transwell migration assays}

The cell migration analysis was carried out using a 24-well Transwell chamber system (Costar 3422, Corning Inc, NY, USA). After treatment, A549 cells was cultured into the upper transwell chamber in serum-free DMEM medium. The cells were allowed to migrate for $24 \mathrm{~h}$ toward the lower chamber containing $0.8 \mathrm{ml}$ of medium supplemented with $10 \%$ fetal bovine serum. Afterwards, the migrated cells on the lower surface were stained with $0.4 \%$ Crystal Violet. A549 cells migration capacity was determined by the numbers of cells that migrated through the membranes. The images were randomly captured under microscope magnification $(20 \times)$.

\section{miR-10a mimic and inhibitor transfection}

A549 cells were transfected with equal amount of miR-10a mimics, miR-10a mimics negative control (NC), miR-10a inhibitor, and miR-10a inhibitor NC by using Lipofectamine 2000 (Invitrogen) according to the manufacturer's instruction. The miR-10a mimics, and miR-10a mimics NC used in this study were designed and synthesized by RiboBio (Guangzhou, China). The miR-10a inhibitors and NC were synthesized by GenePharma Corporation (Shanghai GenePharma Co, Ltd, Shanghai, China). After 48 h transfection, cells were harvested for cell proliferation, apoptosis, migration and invasion.

\section{Realtime PCR}

After different treatment, microRNAs were extracted from A549 cells using a mirVana ${ }^{\mathrm{TM}}$ miRs isolation kit (Ambion). miRs-specific reverse transcription primers (RiboBio Co. Ltd) were used to synthesis cDNA. Real-time PCR amplification was conducted by using Applied Biosystems ${ }^{\circledast} 7300$ Real-Time PCR Systems according to the manufacturer's instruction. The primers for miRs amplification were also 
designed by RiboBio Co. Ltd. The amount of each miR was quantified by the comparative CT method using U6 as the loading control.

\section{miR target prediction and Luciferase assay}

The potential targets of miR-10a were predicted using the algorithms TargetSan (http://www.targetscan.org/). The 3' untranslated region (UTR) sequences of human PTEN mRNA and the 3'UTR of PTEN with a mutant at the miR10a binding site were cloned into p-MIR reporter plasmid (Ambion). HEK293 cells were co-transfected with $100 \mathrm{ng}$ p-MIR- PTEN 3'-UTR/p-MIR- PTEN 3'-UTR mut and 10 pmol miR-10a mimics/NC by Lipofectamine 2000. After 36 hours, cells were collected and detected the Firefly and Renilla luciferase activity using the dual-luciferase reporter assay system gene assay kit (Promega, Beijing, China) according to the manufacturer's instructions. Briefly, after washed with PBS, cells were lysed with Passive Lysis Buffer. $20 \mathrm{ml}$ of cell lysate was added into the luminometer plate containing Luciferase Assay Reagent II. The plate was placed in the luminometer, and initiate reading. Then the plate was removed from the luminometer, and added $100 \mu$ of Stop
\& Glo Reagent and placed in the luminometer and read the value again.

\section{Western blot}

Cells were seeded and treated in 6-wells plates. After treatment, cells were harvested and lysed using RIPA lysis buffer. The concentration of protein was measured using a BCA protein assay. $20 \mathrm{mg}$ total protein in each group was loaded into a SDS-PAGE gel, and then transferred to a PVDF membrane. After blocking with 5\% skim milk, the membrane was incubated with the primary antibodies at $4^{\circ} \mathrm{C}$ overnight. After incubation with HRP-labeled secondary antibody, ECL was used to detect the protein signal. b-actin was used as a loading control.

\section{Statistical analysis}

The data were expressed as mean \pm standard deviation. Student's $t$-test was used for comparison between two groups, and a one-way ANOVA was for multiple groups. Statistical analysis was performed with SPSS 17.0 statistical software, and $p$-value $<0.05$ was interpreted as being statistically significant.
A

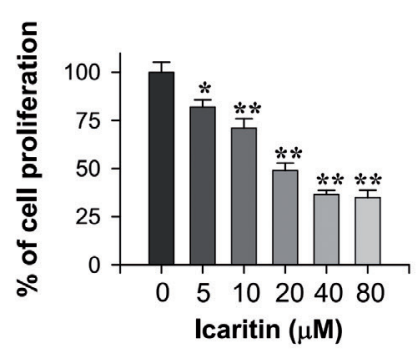

B

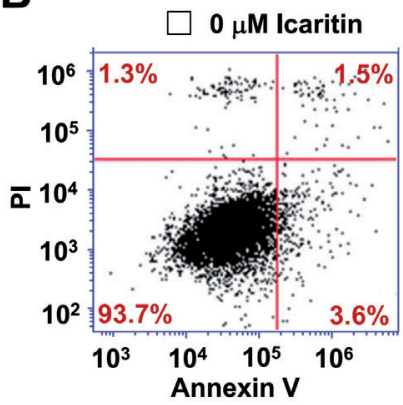

$40 \mu \mathrm{M}$ Icaritin

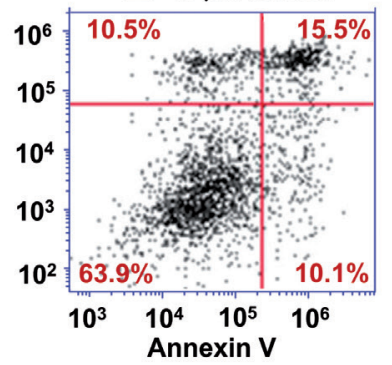

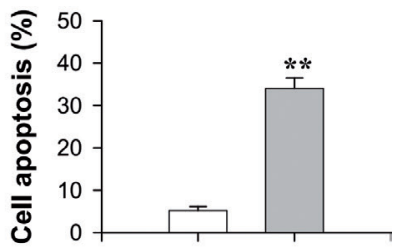

\section{C}
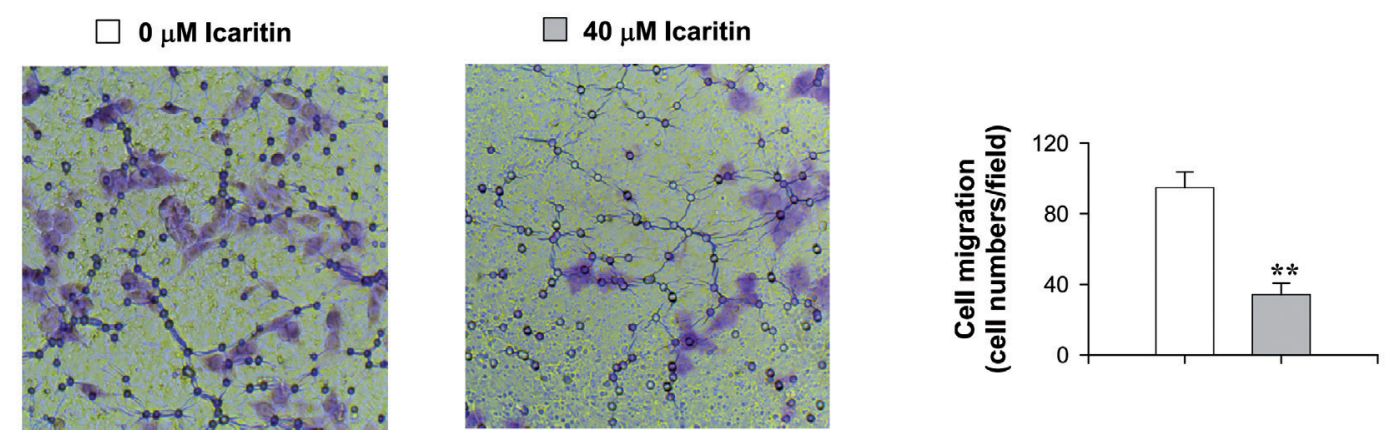

Figure 1. Icaritin suppresses cell growth, induces apoptosis and inhibits migration in A549 cancer cells. A. MTT results showing the level of cell proliferation $(n=5)$. B. Left: typically data in flow cytometry in 0 and $40 \mathrm{mM}$ icaritin groups. Right: the averaged rate of apoptotic cells indicated by the upper and lower right quadrant areas in left panel $(n=5)$. C. Left: representative imaging of cell migration in transwell assay, 20x. Right: quantification and comparison of A549 cell migration capacity, defined by the numbers per field after $24 \mathrm{~h}$ in transwell assay $(n=6) .{ }^{*} p<0.05 v s .0 \mathrm{mM}$ icaritin group and ${ }^{* *} p<0.01 v s .0 \mathrm{mM}$ icaritin group. 


\section{Results}

Icaritin suppresses cell growth, induces apoptosis and inhibits migration in human A549 lung cancer cells

To optimize and establish the anti-tumor model of icaritin, human A549 lung cancer cells were treated with different concentrations of icaritin $(5,10,20,40$ and $80 \mathrm{mM})$ for $48 \mathrm{~h}$ at $37^{\circ} \mathrm{C}$. MTT assay was used to measure the cell growth. As shown in Figure 1A, icaritin effectively suppressed the cell proliferation of A549 in a dose-dependent manner. The inhibitory effect of icaritin on A549 cells began at $5 \mathrm{mM}$ and reached to peak value at $40 \mathrm{mM}$ icaritin treatment. Therefore, we chose $40 \mathrm{mM}$ icaritin for the following experiments. To determine whether icaritin-induced suppression of cell growth is due to cell apoptosis, we measured the rate of cell apoptosis by using annexin-PI staining combined with flow cytometry. We found that treatment with $40 \mathrm{mM}$ icaritin greatly induced cell apoptosis (Fig. 1B), agree well with previous findings (Zheng et al. 2014).

Next, we used transwell migration assay to examine the effect of icaritin on cell migration and invasion in A549 cells. As shown in Figure 1C, the cell migration ability, de-

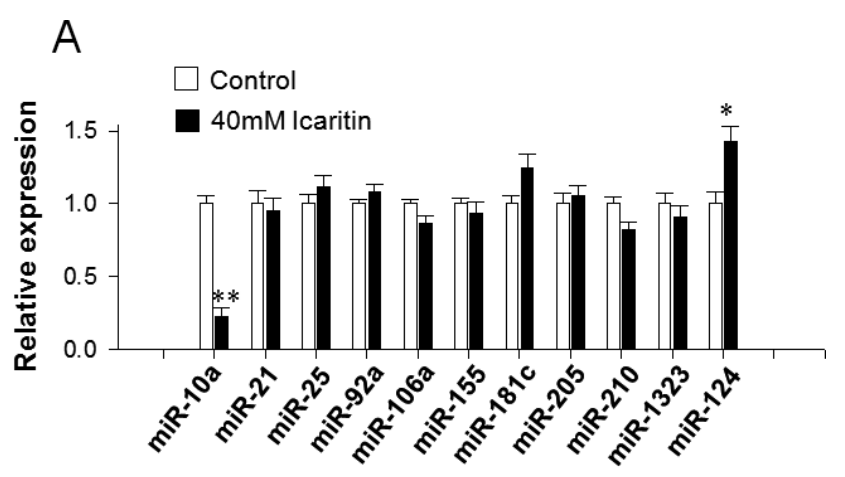

B

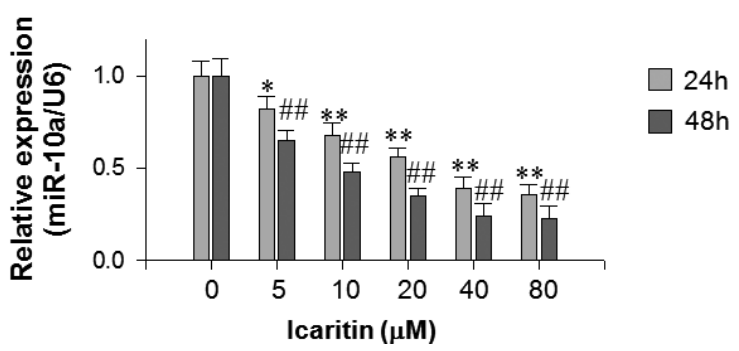

Figure 2. miR-10a is down-regulated after icaritin treatment in A549 cells. A. Realtime PCR results showing the expression level change of oncogenetic miRs after $40 \mathrm{mM}$ icaritin treatment. ${ }^{* *} p<0.01 v s .0 \mathrm{mM}$ icaritin group $(n=5)$. B. The expression level change of miR-10a after $24 \mathrm{~h}$ or $48 \mathrm{~h}$ treatment with different concentration of icaritin $(n=3)$. ${ }^{*} p<0.05$ vs. $0 \mathrm{mM}$ icaritin in 24 h group and ${ }^{* *} p<0.01 v s .0 \mathrm{mM}$ icaritin in 24 h group; ${ }^{\# \#} p<0.01$ vs. $0 \mathrm{mM}$ icaritin in $48 \mathrm{~h}$ group. termined by the numbers of cells that migrated through the membranes after $24 \mathrm{~h}$, was significantly compromised by $40 \mathrm{mM}$ icaritin treatment. Altogether, the above results demonstrated an anti-tumor effect of icaritin on cell proliferation, apoptosis, cell migration in human A549 lung cancer cells.

\section{miR-10a is down-regulated after icaritin treatment in hu-} man A549 lung cancer cells

To explore the potential role of miRs in the anti-tumor effect of icaritin in A549 cancer cells, we first performed real-time PCR to detect the expression level change of oncogenetic miRs, such as miR-10a, miR-21, miR-25, miR-92a, miR-106a, miR-155, miR-181c, miR-205, miR-210 and miR-1323 (Lu et al. 2018). As shown in Figure 2A, the expression level of miR-10a was dramatically down-regulated to $23 \%$ after $40 \mathrm{mM}$ icaritin treatment, but no change was observed in other oncogenetic miRs. Moreover, miR-124 was up-regulated in A549 cells after icaritin treatment, consistent with other's report in human carcinoma cells (Jin et al. 2017). To further determine how the expression level change of miR-10a related to icaritin treatment, human A549 lung cancer cells were exposed to different concentrations of icaritin for $24 \mathrm{~h}$ and $48 \mathrm{~h}$ at $37^{\circ} \mathrm{C}$. We found that treatment with icaritin at $24 \mathrm{~h}$ and $48 \mathrm{~h}$ both significantly down-regulated miR-10a expression, and the inhibitory effect of icaritin showed a dose- and time-dependent manner (Fig. $2 \mathrm{~B}$ ), indicating that the change in the miR-10a expression was strongly relative to the treatment of icaritin.

Over-expression of $\mathrm{miR}-10 \mathrm{a}$ dramatically abrogates the anti-tumor effects of icaritin in A549 cells

Next, we investigated whether down-regulation of miR-10a regulated the anti-tumor effect of icaritin in A549 cancer cells. As shown in Figure 3, compared to the negative control group (NC group), miR-10a mimics greatly reduced the inhibitory effect of icaritin in cell growth (Fig. 3A), and significantly diminished icaritin-induced cell apoptosis (Fig. 3B). Consistent with these findings, transwell migration assay showed that the inhibitory effect of icaritin on cell migration in A549 cells was also partly eliminated by miR-10a overexpression, but not in miR-10a mimic NC group (Fig. 3C). These results demonstrated that over-expression of miR-10a in A549 cells dramatically abrogated the anti-tumor effects of icaritin on cell proliferation, apoptosis, migration, indicating that down-regulation of miR-10a mediated the anti-tumor effects of icaritin in A549 cells.

Suppression of miR-10a reproduces the anti-tumor effects of icaritin in A549 cells

To further investigate whether suppression of miR-10a alone is sufficient to reproduce the anti-tumor effects of icaritin 

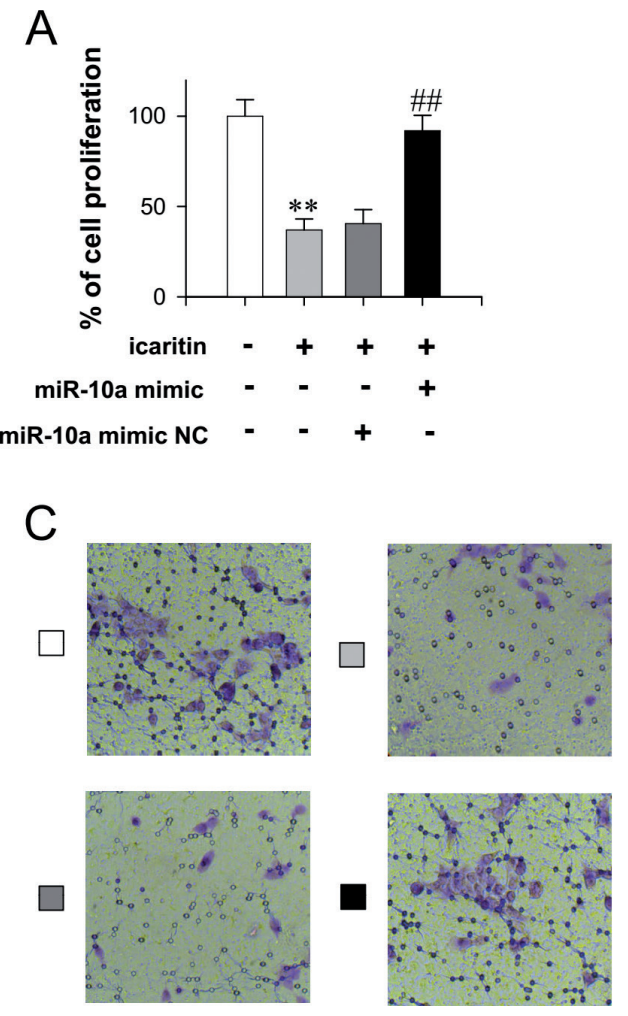

B

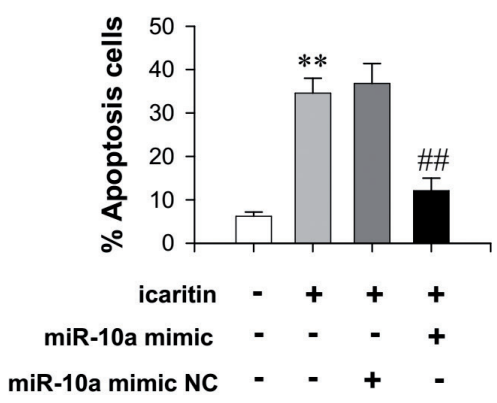

Figure 3. Over-expression of miR-10a dramatically abrogates the anti-tumor effects of icaritin in A549 cells. A. Cell proliferation evaluated by MTT assay after miR-10a mimic treatment. B. The averaged rate of apoptotic cells after miR-10a mimic treatment. C. Left: representative imaging of cell migration in transwell assay, 20x. Right: quantification and comparison of A549 cell migration after $24 \mathrm{~h}(n=6) .{ }^{* *} p<0.01$ vs. $0 \mathrm{mM}$ icaritin group, ${ }^{\# \#} p<0.01$ vs. 40 $\mathrm{mM}$ icaritin+miR-10a mimic NC group. in A549 cells, we used miR-10a inhibitor to suppress miR10a expression in A549 cells. Real-time PCR result showed that compared with miR-10a inhibitor NC group, miR$10 \mathrm{a}$ inhibitor greatly reduced miR-10a expression level to about 25\% (Fig. 4A), which was comparable with $40 \mathrm{mM}$ icaritin treatment (Fig. 2A). MTT and cell apoptosis assay demonstrated that suppression of miR-10a by miR-10a inhibitor significantly suppressed cell proliferation (Fig. 4B), and greatly induced cell apoptosis in A549 cells (Fig. 4C). Moreover, the effect of miR-10a down-regulation in A549 cells was also evaluated using transwell migration assay. Expectedly, miR-10a inhibitor dramatically induced cell
A

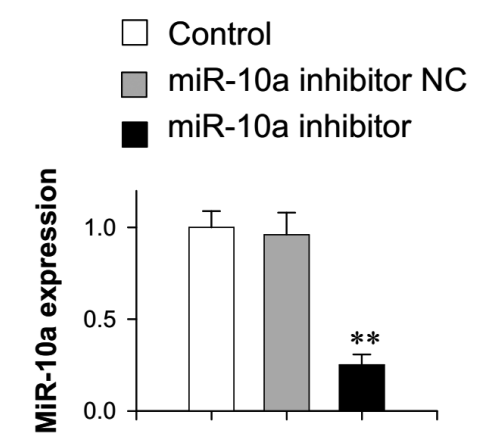

C

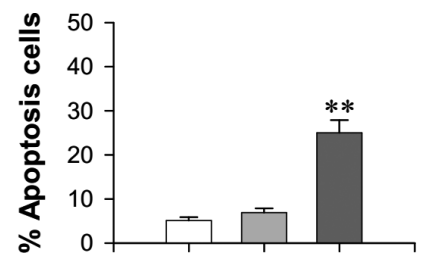

\section{B}

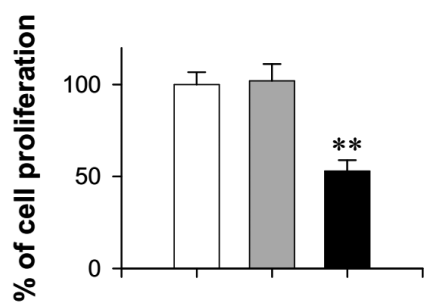

D

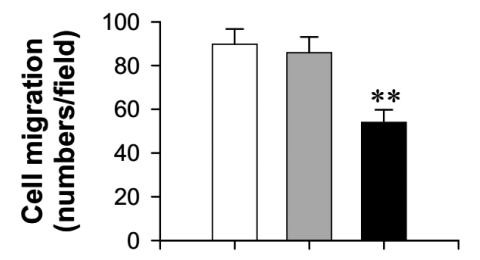

Figure 4. Suppression of miR-10a reproduces the anti-tumor effects of icaritin in A549 cells. A. Realtime PCR results showing the expression level of miR-10a $(n=5)$. B. MTT assay showing the rate of cell proliferation after suppression of miR-10a $(n=5)$. C. The averaged rate of apoptotic cells after suppression of miR-10a $(n=4)$. D. Quantification and comparison of A549 cell migration after $24 \mathrm{~h}$ in transwell assay $(n=4) .{ }^{* *} p<0.01$ vs. miR-10a inhibitor NC group. 
migration after $48 \mathrm{~h}$ incubation (Fig. 4D), indicating that suppression of miR-10a expression alone in A549 cells is sufficient to reproduce the anti-tumor effect of icaritin on cell proliferation, apoptosis, migration and invasion.

PTEN is a direct target of miR-10a and mediates the antitumor effects of icaritin in A549 cells

Next, we performed bioinformatics analysis to predict the direct target of miR-10a using algorithms TargetSan. The result showed that miR-10a has a direct binding site in the 3'- UTR of PTEN mRNA (Fig. 5A). Luciferase reporter assay demonstrated that miR-10a mimic significantly diminished the luciferase activity compared with NC group (Fig. 5B). However, mutating the putative miR-10 binding site by replacing the AGGG with UCCC (Fig. 5A) effectively eliminated the inhibition of luciferase activity by miR-10 mimic (Fig. 5B), indicating that PTEN is one of the targets of miR-10a. In fact, Western blot result showed that the expression level of PTEN in A549 cells after icaritin treatment was greatly elevated (Fig. 5C), which was negatively correlated with miR-10a (Fig. 2), suggesting that miR-10a targets to PTEN to mediate anti-tumor effect of icaritin in A549 cells.
PI3K/AKT and ERK1/2 pathways are known to be regulated by PTEN, and are associated with cell survival, proliferation, and migration (Chalhoub and Baker 2009). To determine whether PI3K/AKT and ERK1/2 pathways involved in the anti-tumor effect of icaritin, the phosphorylation level of AKT (p-AKT) and ERK1/2 (p-ERK) were measured by Western blot analysis. As shown in Figure $5 \mathrm{C}$, the levels of $\mathrm{p}-\mathrm{AKT}$ and $\mathrm{p}$-ERK were dramatically reduced after icaritin treatment, while there is no change in the total protein levels of AKT and ERK. In addition, miR-10a mimics greatly increase the levels of p-AKT and p-ERK, mainly by elevating the expression levels of PTEN. These results indicated that miR-10a mediates the anti-tumor effects of icaritin via the PTEN/AKT and ERK pathway.

\section{Discussion}

In the present study, to explore the potential roles of miRs involving in the anti-tumor effects of icaritin in A549 cells, we performed real-time PCR to screen the expression level change of oncogenetic miRs, and found that the expression level of miR-10a was well-correlated with the exposed time
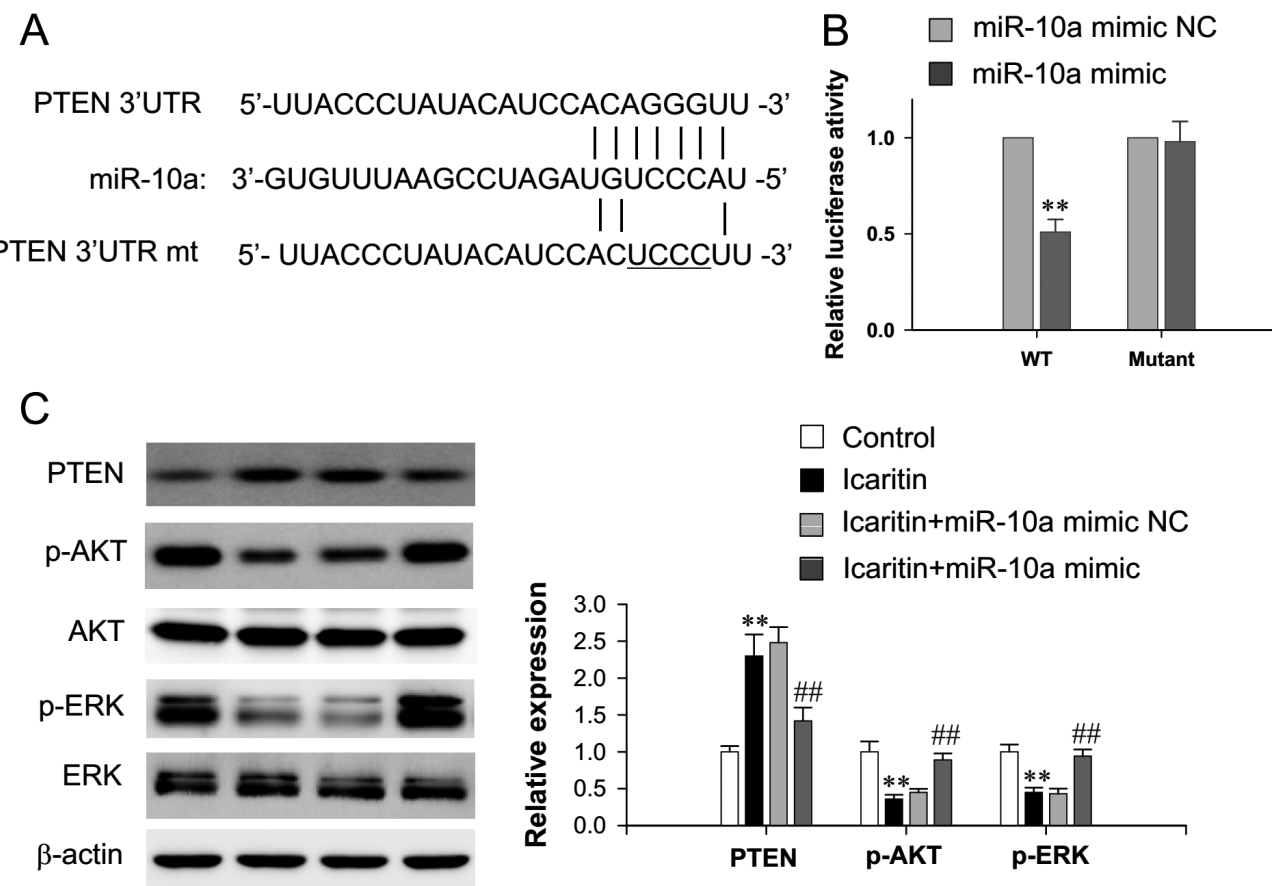

Figure 5. miR-10a mediated the anti-tumor effects of icaritin via the PTEN/AKT/ERK pathway by directly targeting to PTEN. A. A diagram showing miR-10a has a direct binding site in the 3'-UTR of PTEN mRNA. mt: mutation, mutating the putative miR-10 binding site by replacing the AGGG with UCCC. B. Luciferase reporter assay showing miR-10a mimic significantly diminished the luciferase activity compared with NC group $(n=4)$. C. Representative Western blot imaging (left) and the averaged data (right) showing the expression level of PTEN and the phosphorylation level of Akt and ERK after $40 \mathrm{mM}$ icaritin treatment and miR-10a mimic transfection $(n=5)$. ${ }^{* *} p<0.01$ vs. $0 \mathrm{mM}$ icaritin group, ${ }^{\# \#} p<0.01$ vs. $40 \mathrm{mM}$ icaritin+miR-10a mimic NC group. 
and concentration of icaritin. Gain and loss of function experiments showed that down-regulation of miR-10a in A549 cells mediates the anti-tumor effects of icaritin on cell proliferation, apoptosis and migration, which provides a novel insight into the anti-tumor mechanism of icaritin and may be used as a new strategy for lung cancer therapy.

Icaritin is prenylflavonoid derivative from Epimedium Genus that has long been used as a Chinese traditional medicine. Previous studies demonstrated that icaritin exhibits multiple kinds of pharmacological and biological activities, including neuronal protection against myloid-induced neurotoxicity (Wang et al. 2007), induction of embryonic stem cells differentiation into cardiomyocytes (Zhu and Lou 2005), suppression of steroid-associated osteonecrosis and osteoclastic differentiation (Huang et al. 2007; Chen et al. 2018), self-renewal of mouse embryonic stem cells (Tsang et al. 2017), and improvement of hematopoietic function (Sun et al. 2018). Recently, icaritin has been shown an antitumor effect of icaritin in various solid tumors, such as lung cancer (Zheng et al. 2014), hepatocellular carcinoma (Sun et al. 2015; Hu et al. 2016; Lu et al. 2017), breast cancer (Guo et al. 2011; Tiong et al. 2012), esophageal cancer (Han et al. 2018), and glioblastoma (Li et al. 2018; Liu et al. 2018). Except for the studies of icaritin on the solid tumors, icaritin has also been demonstrated a significant inhibitory effect on multiple hematological cancer cells, including leukemia, lymphoma and multiple myeloma (Zhu et al. 2011; Li et al. 2013; Yang et al. 2019). It has been reported that in human NSCLC A549 cells, icaritin inhibit cancer cell growth by inducing cell cycle arrest at $S$ phase and promote cell apoptosis (Zheng et al. 2014). The inhibitor effects of icaritin mentioned above were mediated by the complex crosstalk between different cell signaling pathways, including PI3K/ AKT, MAPKs, and JAK/STATs, which ultimately regulate the cell growth, proliferation, migration and diferentiation. Agree well with these studies, here we also found that treatment human NSCLC A549 cells with icaritin significantly suppressed cell proliferation and induced cell apoptosis in a dose-dependent manner.

Vairous of miRs have been shown to be involved in cell proliferation, differentiation, and tumorigenesis in NSCLC A549 cells (Lu et al. 2018). It has been reported that miR$10 \mathrm{a}$ is upregulated in NSCLC and may promote cancer by targeting PTEN (Yu et al. 2015). miR-21 also targets to PTEN to promote proliferation of NSCLC (Shen et al. 2014). In addition, miR-92a, miR-106a and miR-205 can also bind to PTEN and exert similar effects in NSCLC (Lei et al. 2013; Xie et al. 2015; Ren et al. 2016). miR-155, an important oncogenic miR in vivo, target the suppressor of cytokine signaling 1 gene to activate Akt signaling pathway and promote cancer growth (Xu et al. 2013). miR-25 can reduce the sensitivity to radiation by binding to the B-cell translocation gene 2 in NSCLC (He et al. 2015). miR-210 and miR-1323 also weaken the radiosensitivity of NSCLC cells by repairing genomic double-strand breaks induced by radioation and by promoting the expression of protein kinase, DNA-activated, catalytic polypeptide in NSCLC (Grosso et al. 2013; Li et al. 2015). Consistent with other's report in human carcinoma cells (Jin et al. 2017), miR-124 was up-regulated in A549 cells after icaritin treatment. By screening, we found miR-10a is the only oncogenetic miR that is down-regulation after icaritin treatment. miR-10a mimic greatly eliminated the anti-tumor effect of icaritin cell proliferation, apoptosis and migration, indicating that down-regulation of miR-10a mediates the anti-tumor effect of icaritin in NSCLC A549 cells. We further identified that one of miR-10a's targets, PTEN, also involved in the the anti-tumor effect of icaritin. PTEN is classically associated with inhibiting the PI3K/AKT signal pathway, and ultimately suppresses cell survival, proliferation, and migration (Chalhoub and Baker 2009). Here expectedly, the levels of p-AKT and p-ERK were dramatically reduced after icaritin treatment, which is mainly by elevating the expression levels of PTEN, suggesting that miR-10a mediates the anti-tumor effectos of icaritin via the PTEN/AKT and ERK pathway.

In conclusion, we found that down-regulation of miR-10a in A549 cells mediates the anti-tumor effects of icaritin on cell proliferation, apoptosis and migration via the PTEN/ AKT and ERK pathway, which provides provide novel strategies to practical clinical problems.

Acknowledgments. This work was supported by Health and Family Planning Commission of Wuxi city, China (Grant No. MS201601).

Conflict of interest. The authors have declared no conflict of interest.

\section{References}

Bray F, Ferlay J, Soerjomataram I, Siegel RL, Torre LA, Jemal A (2018): Global cancer statistics 2018: GLOBOCAN estimates of incidence and mortality worldwide for 36 cancers in 185 countries. CA Cancer J. Clin. 68, 394-424 https://doi.org/10.3322/caac.21492

Buttery RC, Rintoul RC, Sethi T (2004): Small cell lung cancer: the importance of the extracellular matrix. Int. J. Biochem. Cell Biol. 36, 1154-1160 https://doi.org/10.1016/S1357-2725(03)00261-9

Caramori G, Casolari P, Cavallesco GN, Giuffre S, Adcock I, Papi A (2011): Mechanisms involved in lung cancer development in COPD. Int. J. Biochem. Cell Biol. 43, 1030-1044 https://doi.org/10.1016/j.biocel.2010.08.022

Chalhoub N, Baker SJ (2009): PTEN and the PI3-kinase pathway in cancer. Ann. Rev. Pathol. 4, 127-150 https://doi.org/10.1146/annurev.pathol.4.110807.092311

Chen S, Zheng L, Zhang J, Wu H, Wang N, Tong W, Xu J, Huang L, Zhang Y, Yang Z, et al. (2018): A novel bone targeting de- 
livery system carrying phytomolecule icaritin for prevention of steroid-associated osteonecrosis in rats. Bone 106, 52-60 https://doi.org/10.1016/j.bone.2017.09.011

Cho JH (2017): Immunotherapy for non-small-cell lung cancer: current status and future obstacles. Immune Netw. 17, 378-391 https://doi.org/10.4110/in.2017.17.6.378

Grosso S, Doyen J, Parks SK, Bertero T, Paye A, Cardinaud B, Gounon P, Lacas-Gervais S, Noel A, Pouyssegur J, et al. (2013): MiR-210 promotes a hypoxic phenotype and increases radioresistance in human lung cancer cell lines. Cell Death Dis. 4, e544 https://doi.org/10.1038/cddis.2013.71

Guo Y, Zhang X, Meng J, Wang ZY (2011): An anticancer agent icaritin induces sustained activation of the extracellular signalregulated kinase (ERK) pathway and inhibits growth of breast cancer cells. Eur. J. Pharmacol. 658, 114-122 https://doi.org/10.1016/j.ejphar.2011.02.005

Han S, Gou Y, Jin D, Ma J, Chen M, Dong X (2018): Effects of Icaritin on the physiological activities of esophageal cancer stem cells. Biochem. Biophys. Res. Commun. 504, 792-796 https://doi.org/10.1016/j.bbrc.2018.08.060

Hayes J, Peruzzi PP, Lawler S (2014): MicroRNAs in cancer: biomarkers, functions and therapy. Trends Mol. Med. 20, 460-469 https://doi.org/10.1016/j.molmed.2014.06.005

He Z, Liu Y, Xiao B, Qian X (2015): miR-25 modulates NSCLC cell radio-sensitivity through directly inhibiting BTG2 expression. Biochem. Biophys. Res. Commun. 457, 235-241 https://doi.org/10.1016/j.bbrc.2014.12.094

Hu J, Zhu W, Wei B, Wen H, Mao S, Xu H, Hu M, Yang T, Jiang H (2016): Antitumoral action of icaritin in LNCaP prostate cancer cells by regulating PEA3/HER2/AR signaling. Anticancer Drugs 2, 944-952 https://doi.org/10.1097/CAD.0000000000000420

Huang J, Yuan L, Wang X, Zhang TL, Wang K (2007): Icaritin and its glycosides enhance osteoblastic, but suppress osteoclastic, differentiation and activity in vitro. Life Sci. 81, 832-840 https://doi.org/10.1016/j.lfs.2007.07.015

Jin L, Miao J, Liu Y, Li X, Jie Y, Niu Q, Han X (2017): Icaritin induces mitochondrial apoptosis by up-regulating miR-124 in human oral squamous cell carcinoma cells. Biomed. Pharmacother. $\mathbf{8 5}, 287-295$ https://doi.org/10.1016/j.biopha.2016.11.023

Lei L, Huang Y, Gong W (2013): miR-205 promotes the growth, metastasis and chemoresistance of NSCLC cells by targeting PTEN. Oncol. Rep. 30, 2897-2902 https://doi.org/10.3892/or.2013.2755

Li H, Liang Q, Wang L (2018): Icaritin inhibits glioblastoma cell viability and glycolysis by blocking the IL-6/Stat3 pathway. J. Cell. Biochem. 120, 7257-7264 https://doi.org/10.1002/jcb.28000

Li Q, Huai L, Zhang C, Wang C, Jia Y, Chen Y, Yu P, Wang H, Rao Q, Wang M, Wang J (2013): Icaritin induces AML cell apoptosis via the MAPK/ERK and PI3K/AKT signal pathways. Int. J. Hematol. 97, 617-623 https://doi.org/10.1007/s12185-013-1317-9

Li Y, Han W, Ni TT, Lu L, Huang M, Zhang Y, Cao H, Zhang HQ, Luo W, Li H (2015): Knockdown of microRNA-1323 restores sensitivity to radiation by suppression of PRKDC activity in radiation-resistant lung cancer cells. Oncol. Rep. 33, 2821-2828 https://doi.org/10.3892/or.2015.3884

Li ZJ, Yao C, Liu SF, Chen L, Xi YM, Zhang W, Zhang GS (2014): Cytotoxic effect of icaritin and its mechanisms in inducing apoptosis in human burkitt lymphoma cell line. Biomed. Res. Int. 2014, 391512

https://doi.org/10.1155/2014/391512

Lin SS, Lai KC, Hsu SC, Yang JS, Kuo CL, Lin JP, Ma YS, Wu CC, Chung JG (2009): Curcumin inhibits the migration and invasion of human A549 lung cancer cells through the inhibition of matrix metalloproteinase-2 and - 9 and Vascular Endothelial Growth Factor (VEGF). Cancer Lett. 285, 127-133 https://doi.org/10.1016/j.canlet.2009.04.037

Liu Q, Zhang H, Jiang X, Qian C, Liu Z, Luo D (2017): Factors involved in cancer metastasis: a better understanding to „seed and soil“ hypothesis. Mol. Cancer 16, 176 https://doi.org/10.1186/s12943-017-0742-4

Liu Y, Shi L, Liu Y, Li P, Jiang G, Gao X, Zhang Y, Jiang C, Zhu W, Han H, Ju F (2018): Activation of PPARgamma mediates icaritin-induced cell cycle arrest and apoptosis in glioblastoma multiforme. Biomed. Pharmacother. 100, 358-366 https://doi.org/10.1016/j.biopha.2018.02.006

Lu J, Zhan Y, Feng J, Luo J, Fan S (2018): MicroRNAs associated with therapy of non-small cell lung cancer. Int. J. Biol. Sci. 14, 390-397 https://doi.org/10.7150/ijbs.22243

Lu PH, Chen MB, Liu YY, Wu MH, Li WT, Wei MX, Liu CY, Qin SK (2017): Identification of sphingosine kinase 1 (SphK1) as a primary target of icaritin in hepatocellular carcinoma cells. Oncotarget 8, 22800-22810 https://doi.org/10.18632/oncotarget.15205

Molina JR, Yang P, Cassivi SD, Schild SE, Adjei AA (2008): Nonsmall cell lung cancer: epidemiology, risk factors, treatment, and survivorship. Mayo Clin. Proc. 83, 584-594 https://doi.org/10.1016/S0025-6196(11)60735-0

Peng Y, Croce CM (2016): The role of MicroRNAs in human cancer. Signal Transduct. Target. Ther. 1, 15004 https://doi.org/10.1038/sigtrans.2015.4

Ren P, Gong F, Zhang Y, Jiang J, Zhang H (2016): MicroRNA-92a promotes growth, metastasis, and chemoresistance in non-small cell lung cancer cells by targeting PTEN. Tumour Biol. 37, 3215-3225 https://doi.org/10.1007/s13277-015-4150-3

Seyfried TN, Huysentruyt LC (2013): On the origin of cancer metastasis. Crit. Rev. Oncog. 18, 43-73 https://doi.org/10.1615/CritRevOncog.v18.i1-2.40

Shen H, Zhu F, Liu J, Xu T, Pei D, Wang R, Qian Y, Li Q, Wang L, et al. (2014): Alteration in Mir-21/PTEN expression modulates gefitinib resistance in non-small cell lung cancer. PloS One 9, e103305 https://doi.org/10.1371/journal.pone.0103305

Sun C, Yang J, Pan L, Guo N, Li B, Yao J, Wang M, Qi C, Zhang G, Liu Z (2018): Improvement of icaritin on hematopoietic function in cyclophosphamide-induced myelosuppression mice. Immunopharmacol. Immunotoxicol. 40, 25-34 https://doi.org/10.1080/08923973.2017.1392564

Sun H, Gao D (2018): Propofol suppresses growth, migration and invasion of A549 cells by down-regulation of miR-372. BMC Cancer 18, 1252 https://doi.org/10.1186/s12885-018-5175-y 
Sun L, Peng Q, Qu L, Gong L, Si J (2015): Anticancer agent icaritin induces apoptosis through caspase-dependent pathways in human hepatocellular carcinoma cells. Mol. Med. Rep. 11, 3094-3100 https://doi.org/10.3892/mmr.2014.3007

Tiong CT, Chen C, Zhang SJ, Li J, Soshilov A, Denison MS, Lee LS, Tam VH, Wong SP, Xu HE, Yong EL (2012): A novel prenylflavone restricts breast cancer cell growth through AhRmediated destabilization of ERalpha protein. Carcinogenesis 33, 1089-1097 https://doi.org/10.1093/carcin/bgs110

Torre LA, Bray F, Siegel RL, Ferlay J, Lortet-Tieulent J, Jemal A (2015) Global cancer statistics, 2012. CA Cancer J. Clin. 65, 87-108 https://doi.org/10.3322/caac.21262

Tsang WP, Zhang F, He Q, Cai W, Huang J, Chan WY, Shen Z, Wan C (2017): Icaritin enhances mESC self-renewal through upregulating core pluripotency transcription factors mediated by ERalpha. Sci. Rep. 7, 40894 https://doi.org/10.1038/srep40894

Wang S, Yan Y, Cheng Z, Hu Y, Liu T (2018): Sotetsuflavone suppresses invasion and metastasis in non-small-cell lung cancer A549 cells by reversing EMT via the TNF-alpha/NF-kappaB and PI3K/AKT signaling pathway. Cell Death Discov. 4, 26 https://doi.org/10.1038/s41420-018-0026-9

Wang Z, Zhang X, Wang H, Qi L, Lou Y (2007): Neuroprotective effects of icaritin against beta amyloid-induced neurotoxicity in primary cultured rat neuronal cells via estrogen-dependent pathway. Neurosci. 145, 911-922

https://doi.org/10.1016/j.neuroscience.2006.12.059

Wu T, Wang S, Wu J, Lin Z, Sui X, Xu X, Shimizu N, Chen B, Wang $\mathrm{X}$ (2015): Icaritin induces lytic cytotoxicity in extranodal NK/Tcell lymphoma. J. Exp. Clin. Cancer Res. CR. 34, 17 https://doi.org/10.1186/s13046-015-0133-X

Xie X, Liu HT, Mei J, Ding FB, Xiao HB, Hu FQ, Hu R, Wang MS (2015): miR-106a promotes growth and metastasis of non-small cell lung cancer by targeting PTEN. Int. J. Clin. Exp. Pathol. 8, 3827-3834

Xu B, Jiang C, Han H, Liu H, Tang M, Liu L, Ji W, Lu X, Yang X, Zhang Y, Liu Y (2015): Icaritin inhibits the invasion and epithelial-to-mesenchymal transition of glioblastoma cells by targeting EMMPRIN via PTEN/AKt/HIF-1alpha signalling. Clin. Exp. Pharmacol. Physiol. 42, 1296-1307 https://doi.org/10.1111/1440-1681.12488

Xu TP, Zhu CH, Zhang J, Xia R, Wu FL, Han L, Shen H, Liu LX, Shu YQ (2013): MicroRNA-155 expression has prognostic value in patients with non-small cell lung cancer and digestive system carcinomas. Asian Pac. J. Cancer Prev. 14, 7085-7090

https://doi.org/10.7314/APJCP.2013.14.12.7085

Yang XJ, Xi YM, Li ZJ (2019): Icaritin: a novel natural candidate for hematological malignancies therapy. BioMed Res. Int. 2019, 4860268 https://doi.org/10.1155/2019/4860268

Yu T, Liu L, Li J, Yan M, Lin H, Liu Y, Chu D, Tu H, Gu A, Yao M (2015): MiRNA-10a is upregulated in NSCLC and may promote cancer by targeting PTEN. Oncotarget 6, 30239-30250 https://doi.org/10.18632/oncotarget.4972

Zappa C, Mousa SA (2016): Non-small cell lung cancer: current treatment and future advances. Transl. Lung Cancer Res. 5, 288-300 https://doi.org/10.21037/tlcr.2016.06.07

Zheng Q, Liu WW, Li B, Chen HJ, Zhu WS, Yang GX, Chen MJ, He GY (2014): Anticancer effect of icaritin on human lung cancer cells through inducing $S$ phase cell cycle arrest and apoptosis. J. Huazhong Univ. Sci. Technolog. Med. Sci. 34, 497-503 https://doi.org/10.1007/s11596-014-1305-1

Zhu DY, Lou YJ (2005): Inducible effects of icariin, icaritin, and desmethylicaritin on directional differentiation of embryonic stem cells into cardiomyocytes in vitro. Acta Pharmacol. Sin. 26, 477-485 https://doi.org/10.1111/j.1745-7254.2005.00076.x

Zhu J, Li Z, Zhang G, Meng K, Kuang W, Li J, Zhou X, Li R, Peng H, Dai C, et al. (2011): Icaritin shows potent anti-leukemia activity on chronic myeloid leukemia in vitro and in vivo by regulating MAPK/ERK/JNK and JAK2/STAT3 /AKT signalings. PloS One. 6, e23720

https://doi.org/10.1371/journal.pone.0023720

Zhu S, Wang Z, Li Z, Peng H, Luo Y, Deng M, Li R, Dai C, Xu Y, Liu S, et al. (2015): Icaritin suppresses multiple myeloma, by inhibiting IL-6/JAK2/STAT3. Oncotarget 6, 10460-10472 https://doi.org/10.18632/oncotarget.3399

Received: July 7, 2019

Final version accepted: September 20, 2019 